\title{
Dermatitis artefacta mimicking cutaneous vasculitis: case report and literature overview
}

\author{
João Victor Vecchi Ferri ${ }^{1}$, Daniel Brito de Araujo ${ }^{2}$ \\ ${ }^{1}$ Department of General Surgery, Hospital de Clínicas de Porto Alegre, Porto Alegre, Brazil \\ ${ }^{2}$ Department of Internal Medicine, Faculty of Medicine, Federal University of Pelotas, Pelotas, Brazil
}

\begin{abstract}
A 31-year-old Caucasian woman with a history of anxiety disorder presented with chronic ulcerative lesions of the skin in arms and legs, treated initially as cutaneous vasculitis, evolving with relapsing during corticosteroid tapering, was diagnosed, after thorough investigation and no organic disease found, with dermatitis artefacta - a self-inflicted harm due to psychological disorders. Dermatitis artefacta is a rare condition, more frequent in women, in which traumatic skin lesions are caused by the patient him/herself, over accessible parts of the body, due to personality disorders. Clinicians should be aware of this alternative aetiology, especially as a differential diagnosis for refractory cutaneous vasculitis.
\end{abstract}

Key words: dermatitis artefacta, vasculitis, factitious disorders.

\section{Introduction}

Dermatitis artefacta (DA), also known as factitious dermatitis, is a rare psychosomatic syndrome in which skin lesions are traumatically self-induced, with many forms of presentation that may involve any part of the body.

Dermatitis artefacta is more frequent in women (female to male ratio of 3-20:1), with higher involvement during adolescence and young adulthood, but it may also occur in older ages, with a broad variable onset of nine to 73 years, especially in emotionally ill patients [1-12].

\section{Case report}

This description refers to a 31-year-old Caucasian female patient with chronic cutaneous ulcerative lesions in legs, arms, and abdomen, which occurred in the past few years. The patient was initially diagnosed with cutaneous vasculitis and treated with prednisone $60 \mathrm{mg} /$ daily, tapered to $10 \mathrm{mg} /$ day in next 10 months, with a good initial response and complete remission of cutaneous lesions. However, as the dose of prednisone reached $10 \mathrm{mg} /$ day, new lesions in arms and legs began to arise, and this time the patient was referred to our department.

On the first evaluation, the patient presented an adequate general health condition, with painful ulcerative lesions in legs (Fig. 1) and arms (Fig. 2), without any further associated symptoms (e.g. fever, weight loss, or other systemic involvement). A medical history of an affective disorder two years before the exam described ulcerative lesions, treated with fluoxetine $40 \mathrm{mg} /$ day.

A complete laboratory evaluation was performed, such as c-ANCA, p-ANCA, antinuclear antibody, complement components level, C-reactive protein, erythrocyte sedimentation rate, blood count, renal and hepatic function tests, hepatitis C, B, and HIV serology, all with normal results. A biopsy of one of the skin wounds showed a fragment with shallow ulceration, with no stratum corneum and only non-specific mild chronic inflammatory process, fibrosis, and capillaries with fibrin thrombus, characterised by well-demarcated borders, suggesting (added to the clinical exam) the diagnosis of DA. The patient was confronted with the biopsy's result and confirmed herself to be responsible for the injuries. Whenever the patient complained of an anxiety crisis, she felt 


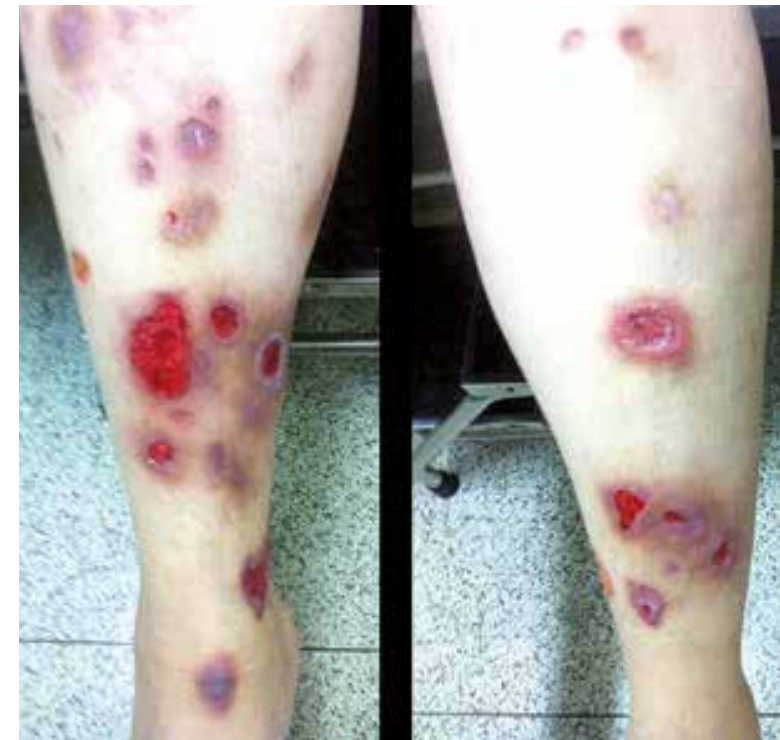

Fig. 1. Multiple skin ulcers in the legs from chronic self-harm and areas of keratinous excoriation from chronic scratching.

an irrepressible need to scratch, mainly the arms and legs, until she was finally able to calm down. During the period of high dosing treatment with prednisone, the anxiety episodes decreased substantially, but as soon as the dose was reduced, the urge to scratch returned.

The patient was referred to a psychiatrist, remaining since then in cognitive-behavioural therapy, with a significant improvement, and in adequate control of the anxiety disorder. Relapse of skin scratching did not occur.

\section{Discussion}

Dermatitis artefacta is a psychological disorder in which an individual self-induces skin lesions, with many forms of presentation, which may involve any part of the body. Bizarre lesions with sharp geometric borders surrounded by normal skin are characteristic of DA.

Skin changes may be the result of various types of damage: mechanical, chemical, thermal, and even electrical, including fingernails, sharp or blunt objects, burning cigarettes, and caustic chemicals. Precipitating factors for DA range from simple anxiety to severe personality disorders, including attention-seeking traits, obsessions, compulsions, and psychotic disturbances. Long-standing cases may be secondary to underlying anxiety or depression, emotional deprivation, an unstable body image, or a personality disorder with borderline features [2].

Histopathological features are non-specific, as our biopsy revealed. Usually it has characteristics of acute but mild inflammation with increased polymorphonuclear leucocytes and scattered erythrocytes. Areas of

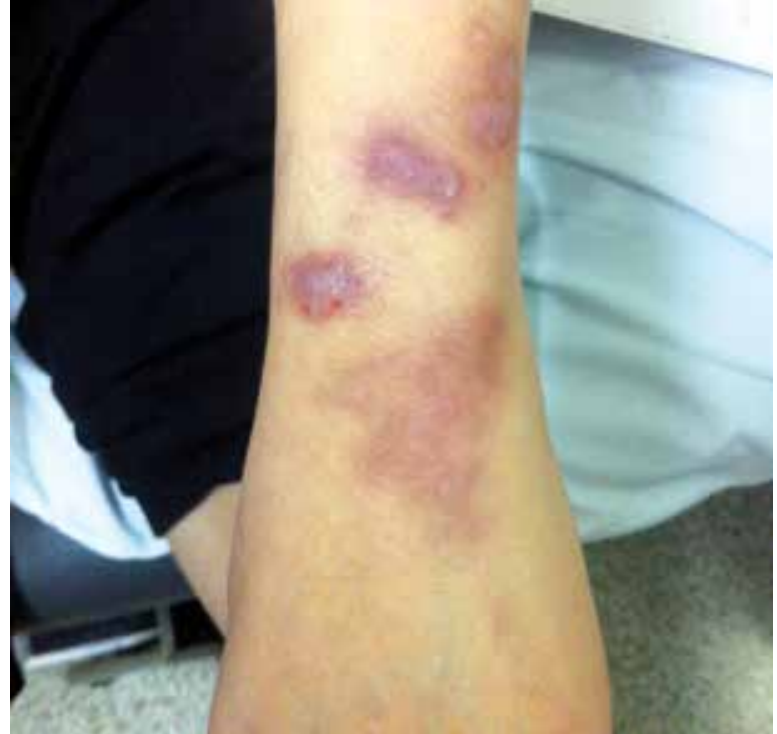

Fig. 2. Old scars in forearm from abrasions caused by scratching.

necrosis with areas of healing and fibrocystic reaction may also occur [3].

The condition must be distinguished from neurotic excoriations and Munchausen's syndrome because the psychopathology, and therefore the treatment, is different in each case. Patients with neurotic excoriations usually have psychiatric comorbidities. The most common comorbidities related to DA are obsessive-compulsive disorder (15-68\%), depression (42\%), and alcohol abuse/dependence (40\%), while those with Munchausen's syndrome are sociopathic personalities [4, 13].

Dermatitis artefacta is more common in women than in men (4.7-7: 1 in patients younger than 16 years, and 3-20: 1 in the general population), but may occur at any age, with the highest frequency during adolescence and young adulthood and in patients that tend to be emotionally immature or have psychosocial or interpersonal difficulties [11, 12]. It may also occur as a cry for help when emotional stresses become too great to endure [5].

In a right-handed person, the left side is usually involved, and the accessible body parts are usually more involved than inaccessible parts like the midline of the dorsum. Recurrent excoriation produces inflammation and lichenification of the skin, resulting in irritation and pruritus, which leads to further self-trauma and chronic dermatitis [1].

The condition tends to wax and wane along with the psychosocial circumstances in the patient's life [6]. Many patients use their lesions to obtain attention, maintain contact with others, and fill their emotional emptiness. The visible skin lesions can be understood 
as an attempt of non-verbal communication sub-serving as an appeal function [6].

According to the latest update of Diagnostic and Statistical Manual of Mental Disorders (DSM-5), diagnostic criteria of DA are based on:

- recurrent skin picking resulting in skin lesions,

- repeated attempts to decrease or stop skin picking,

- the skin picking causes clinically significant distress or impairment in social, occupational, or other important areas of functioning,

- the skin picking is not attributable to the physiological effect of a substance or another medical condition,

- the skin picking is not better explained by symptoms of another mental disorder [7].

When the patient refuses psychiatric referral, the use of psychotropic drugs is helpful and appropriate. High doses of selective serotonin reuptake inhibitors (SSRIs), or low doses of atypical antipsychotic agents, may be effective [4]. Antipsychotic drugs such as risperidone and aripiprazole are the treatment of choice, with an advantage with the latter because of additional antidepressant properties. Pimozide is no longer considered as a firstline therapy because of its extrapyramidal effects [8]. The atypical antipsychotic drug olanzapine appears to be effective in DA therapy [9]. Follow-up studies have shown that most DA patients improve significantly as a result of positive changes in their life situation and during maturation as a result of psychiatric treatment [1].

What is important is that symptoms of DA are improved by treatment with high doses of corticosteroids (CS), which is related to the potential effect of CS as an anxiety reducer, which goes against the general knowledge about their adverse effects. According to Korte [10], these drugs play a central role in anxiety, being able to exercise maladaptive or adaptive effects. The timing of corticosteroid dose increase (before, during, or after exposure to a stressor) determines whether and how behaviour is affected. At high circulating levels, they enhance acquisition, conditioning, and consolidation of an inescapable stressful experience.

The differential diagnoses that could be considered for crusted, blistering lesions include herpes simplex infection, allergic contact dermatitis, porphyria cutanea tarda, epidermolysis bullosa acquisita, amyloidosis, vasculitis, pyoderma gangrenosum, cutaneous lymphoma, drug eruptions, or necrotic cutaneous loxoscelism after a spider bite (genus Loxosceles) [14].

Clinicians should be aware of this alternative aetiology for ulcerative skin lesions, especially when organic conditions such as vasculitis are ruled out but psychological aspects are evident.
The authors declare no conflict of interest.

\section{References}

1. Murray AT, Goble R, Sutton GA. Dermatitis artefacta presenting as a basal cell carcinoma - an important clinical sign missed. Br J Ophthalmol 1998; 82: 97.

2. Gupta AK, Haberman HF. The self-inflicted dermatoses: A critical review. Gen Hosp Psychiatry 1987; 9: 53-57.

3. Antony SJ, Mannion SB. Dermatitis artefacta revisited. Cutis 1995; 55: 362-364.

4. Koblenzer CS. The current management of delusional parasitosis and dermatitis artefacta. Skin Therapy Lett 2010; 15: 1-3.

5. Fabisch W. Psychiatric aspects of dermatitis artefacta. Br J Dermatol 1980; 102: 29-34.

6. Krishna K. Dermatitis artefacta. Indian J Dermatol Venereol Leprol 1995; 61: 178-179.

7. American Psychiatric Association. Diagnostic and Statistical Manual of Mental Disorders, Fifth Edition (DSM-5), American Psychiatric Association, Arlington 2013.

8. Wenning MT, Davy LE, Catalano G, Catalano MC. Atypical antipsychotics in the treatment of delusional parasitosis. Ann Clin Psychiatry 2003; 15: 233-239.

9. Garnis-Jones S. Treatment of self-mutilation with olanzapine. J Cutan Med Surg 2000; 4: 161-163.

10. Korte SM. Corticosteroids in relation to fear, anxiety and psychopathology. Neurosci Biobehav Rev 2001; 25: 117-142.

11. Ehsani, AH, Toosi, S, Mirshams Shahshahani, M, et al. Psychocutaneous disorders: an epidemiologic study. J Eur Acad Dermatol Venereol 2009; 23; 945-947.

12. Koo JYM, Ting TP. Dermatitis artefacta. Elston Medscape, DM (ed.). Available at https://emedicine.medscape.com/article/1121933

13. Mutasim DF, Adams BB. The psychiatric profile of patients with psychogenic excoriation. J Am Acad Dermatol 2009; 61: 611-613.

14. Wong JW, Nguyen TV, Koo JY. Primary psychiatric conditions: dermatitis artefacta, trichotillomania and neurotic excoriations. Indian J Dermatol 2013; 58: 44-48. 